\title{
Dimethyl fumarate accelerates wound healing under diabetic conditions
}

\author{
Ying $\mathrm{Li}^{1}$, Fuzhe $\mathrm{Ma}^{2}$, Huimin $\mathrm{Li}^{3}$, Yuguo Song4,5, Huan Zhang6, Ziping Jiang7 and Hao Wu7,8 \\ 1Department of Dermatology, Affiliated Hospital of Beihua University, Jilin, Jilin, China \\ 2Department of Nephrology, The First Hospital of Jilin University, Changchun, Jilin, China \\ 3Department of Clinical Laboratory, The Second Hospital of Jilin University, Changchun, Jilin, China \\ ${ }^{4}$ Research Institute of Clinical Immunology, Affiliated Hospital of Beihua University, Jilin, Jilin, China \\ ${ }^{5}$ Research Center for Life Sciences, Beihua University, Jilin, Jilin, China \\ ${ }^{6}$ Operating Theater, China-Japan Union Hospital of Jilin University, Changchun, Jilin, China \\ 'Department of Hand and Foot Surgery, The First Hospital of Jilin University, Changchun, Jilin, China \\ ${ }^{8}$ Department of Translational Medicine, The First Hospital of Jilin University, Changchun, Jilin, China \\ Correspondence should be addressed to H Wu or Z Jiang: wuhaobaha@jlu.edu.cn or waterjzp@jlu.edu.cn
}

\begin{abstract}
Impaired wound healing is a common complication among patients with diabetes mellitus (DM), resulting in high rates of disability and mortality. Recent findings highlighted the critical role of nuclear factor erythroid 2-related factor 2 (NRF2) - a master of cellular antioxidants scavenging excessive DM-induced free radicals - in accelerating diabetic wound healing. Dimethyl fumarate (DMF) is a potent NRF2 activator used for the treatment of multiple sclerosis. However, the effect of DMF on wound healing has not been determined. The present study investigated the effect of DMF on the diabetic and the non-diabetic wound healing in streptozotocin-induced diabetic mice and non-diabetic control mice. DMF activated NRF2 signaling under both conditions. Interestingly, DMF attenuated oxidative damage and inflammation and accelerated wound closure in diabetic mice. However, this effect was not observed in non-diabetic mice. Keratinocytes were treated with normal glucose (NG), high glucose (HG) or hydrogen peroxide $\left(\mathrm{H}_{2} \mathrm{O}_{2}\right)$, in the presence or absence of DMF to assess the role of reactive oxygen species (ROS) - inducible in DM - in mediating DMF-induced protection. Both $\mathrm{HG}$ and $\mathrm{H}_{2} \mathrm{O}_{2}$ elevated ROS, oxidative damage and inflammation, the effects of which were similarly blunted by DMF. However, in spite of the activation of NRF2, DMF lost this capability under the NG condition. The findings of this study demonstrate that ROS activate the protective effect of DMF on the diabetic wound healing.
\end{abstract}

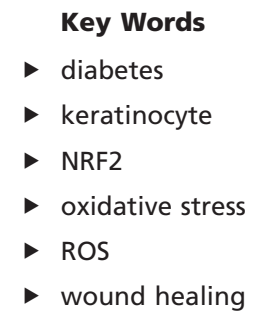

Journal of Molecular Endocrinology (2018) 61, 163-172

\section{Introduction}

Impaired wound healing is a common complication of diabetes mellitus (DM), which remains ineffectively treated. In contrast to normal tissue healing, the diabetic wound displays exceptionally enhanced oxidative stress along with prolonged and intense inflammation (Kampfer et al. 2005, Lan et al. 2013, Davis et al. 2018), which impair the normal process of wound healing. Therefore, blocking oxidative stress or inflammation under diabetic conditions may break this vicious amplifying circle (Hulsmans \& Holvoet 2010), accelerating the diabetic wound healing.

Recent findings highlighted the critical role of nuclear factor erythroid 2-related factor 2 (NRF2) in facilitating 
the diabetic wound healing (Long et al. 2016). NRF2 is a master regulator of cellular antioxidant activity (Kaspar et al. 2009, Zheng et al. 2011). NRF2 transcriptionally activates a series of antioxidant genes, such as heme oxygenase-1 (Ho1) and NAD(P)H dehydrogenase quinone 1 (Nqo1) (Ruiz et al. 2013). NRF2 increased antioxidants function as scavengers for excessive DM-induced free radicals, protecting from oxidative stress-associated cellular injury. NRF2 is pivotal in the prevention of DM-impaired wound healing, since Nrf2 gene-knockout diabetic mice exhibited greater cutaneous oxidative damage and delayed wound closure compared with WT diabetic mice (Long et al. 2016). Moreover, the activation of NRF2 by sulforaphane (SFN) or cinnamaldehyde (CA) significantly alleviated DM-induced oxidative stress and improved the diabetic wound healing (Long et al. 2016). Therefore, the activation of NRF2 holds great potential for the clinical management of diabetic non-healing wounds.

Dimethyl fumarate (DMF) is a potent activator of NRF2 used for the clinical treatment of multiple sclerosis (MS) since 2013 (Linker \& Gold 2013, Bomprezzi 2015). Despite its promising therapeutic effect on MS, the role of DMF in improving the diabetic wound healing has not been determined. Moreover, the effect of NRF2 activation on wound healing under normal blood glucose conditions, covering a broader clinical range of applications in addition to the diabetic wound healing, remains unknown.

The objective of the present study was to investigate the effect of DMF on wound healing under both diabetic and non-diabetic conditions.

\section{Materials and methods}

\section{Animal housing, DM induction and animal treatment}

C57BL/6 mice were housed in the Animal Center of Jilin University, Changchun, China at $22^{\circ} \mathrm{C}$, on a $12: 12$-h lightdarkness cycle with free access to rodent feed and tap water. The Institutional Animal Care and Use Committee of Jilin University approved all the experimental procedures.

Eight-week-old male mice received intraperitoneal injections of either streptozotocin (STZ, $50 \mathrm{mg} / \mathrm{kg} / \mathrm{day}$, dissolved in 0.1 $\mathrm{M}$ sodium citrate, $\mathrm{pH} 4.5$; Sigma-Aldrich) or sodium citrate for five consecutive days (Zheng et al. 2011, Kato et al. 2016, Wu et al. 2016, Sun et al. 2017). One week after the last injection, the fasting glucose levels (4-h fast) were recorded, with a level above $13.89 \mathrm{mM}$ considered diabetic ( $\mathrm{Wu}$ et al. 2016). Immediately after the confirmation of DM, the mice, with their backs shaved and cleaned, were anesthetized by intraperitoneal injection of chloral hydrate at $0.3 \mathrm{mg} / \mathrm{kg}$ (Pan et al. 2017). Subsequently, a sterile $6 \mathrm{~mm}$ skin biopsy punch was used to inflict a single wound on each mouse.

Immediately after the infliction of the wounds, the diabetic mice and control (Ctrl) mice were treated with DMF (MedChem Express, Shanghai, China) by gavage at a series of doses (10, 20 (Garstkiewicz et al. 2017), 40 or $80 \mathrm{mg} / \mathrm{kg}$ ) in $\mathrm{H}_{2} \mathrm{O}$ containing $0.08 \%$ metho-cel and $10 \%$ DMSO (Garstkiewicz et al. 2017) or vehicle alone, to assess the effect of DMF on wound healing under both diabetic and non-diabetic conditions. The horizontal and vertical diameters of each wound were recorded using a digital caliper every other day until 10 days after the onset of DM. The mean of the horizontal and vertical diameters of each wound was calculated. The area of each wound was subsequently calculated and wound healing was expressed as the percentage of the original wound area that had healed (Long et al. 2016). At the end of the procedures, all the mice were euthanized with chloral hydrate, and an $8 \mathrm{~mm}$ skin biopsy punch was used to harvest the skin (Long et al. 2016).

\section{Hematoxylin and eosin (H\&E) and immunohistochemical (IHC) staining}

The freshly harvested cutaneous tissues were fixed immediately into $10 \%$ buffered formalin solution and embedded in paraffin, followed by sectioning into 5 - $\mu$ m-thick sections onto glass slides. H\&E staining was performed to analyze the morphology as previously described (Wu et al. 2014b). IHC staining was performed for the detection of positively stained areas (brick-red) of inflammation, oxidative damage and NRF2/HO1 signaling using a kit provided by MXB Biotechnologies (Fuzhou, Fujian, China). The primary antibodies used for IHC staining were anti-4-hydroxynonenal (4-HNE, Alpha Diagnostic Int., San Antonio, TX, USA; 1:100), anti-HO1 (Santa Cruz Biotechnology; 1:100), anti-NRF2 (Santa Cruz Biotechnology; 1:100) and anti-vascular cell adhesion molecule-1 (VCAM-1, Santa Cruz Biotechnology; 1:100).

\section{Cell culture and experiments}

Keratinocytes (HaCaT cell line) were cultured under normal glucose $(\mathrm{NG}, 5.5 \mathrm{mM})$ condition. The cells were subjected to treatment with DMF at $60 \mu \mathrm{M}$ (Lee et al. $2017)$, in the presence or absence of hydrogen peroxide $\left(\mathrm{H}_{2} \mathrm{O}_{2}, 1 \mathrm{mM}\right)$ or high glucose $(\mathrm{HG}, 25 \mathrm{mM})$, for $6 \mathrm{~h}(\mathrm{Pi}$ et al. 2003) to determine the levels of reactive oxygen 
species (ROS) required to activate the protective effect of DMF. Since $\mathrm{H}_{2} \mathrm{O}_{2}$ is an active form of ROS and has been shown to induce nuclear accumulation of NRF2, it was selected for this test (Pi et al. 2003).

\section{Real-time PCR (RT-PCR)}

Cutaneous tissues or cell lysates of keratinocytes were used for quantitative RT-PCR, as described previously (Wu et al. 2014b). The primers for Gapdh, Ho1, intercellular adhesion molecule-1 (Icam-1), Nqo1 and Vcam-1 were provided by Life Technologies.

\section{Western blot analysis}

Western blot analysis was performed as described previously (Wu et al. 2014b), using cutaneous tissues or cell lysates of keratinocytes. The primary antibodies used were anti-GAPDH (Santa Cruz Biotechnology; 1:3000), anti-HO1 (Santa Cruz Biotechnology, 1:1000), antiICAM-1 (Santa Cruz Biotechnology, 1:500), anti-NQO1 (Santa Cruz Biotechnology, 1:1000), anti-NRF2 (Santa Cruz Biotechnology, 1:1000) and anti-VCAM-1 (Santa Cruz Biotechnology, 1:1000).

\section{Analysis of lipid peroxides and ROS}

Malondialdehyde (MDA) - an indicator of lipid peroxidation - was determined in the cutaneous tissues and cell lysates. The levels of ROS in keratinocytes were determined to evaluate oxidative stress. The levels of MDA and ROS were measured using a lipid peroxidation assay kit and an ROS kit, respectively (Nanjing Jiancheng Bioengineering Institute, Nanjing, Jiangsu, China) according to manufacturer's instructions.

\section{Statistical analysis}

Six mice per group were studied. The cell experiments were performed in triplicate. The measurements for each group were summarized as means \pm s.D. The Image Quant 5.2 software (GE Healthcare Bio-Sciences, Pittsburgh, PA, USA) was used to analyze Western blots. For IHC staining, positively stained areas of the epidermis, excluding the sebaceous gland, were quantified. One-way ANOVA was performed for comparisons among different groups, followed by post hoc pairwise comparisons using Tukey's test with Origin 8.6 data analysis and graphing software Lab (OriginLab, Northampton, MA, USA). A $P<0.05$ denoted statistical significance.

\section{Results}

\section{DMF accelerated wound closure under} diabetic conditions

The diabetic and non-diabetic mice were treated with DMF at different doses $(10,20,40$ or $80 \mathrm{mg} / \mathrm{kg})$ or vehicle to investigate the effect of DMF on wound healing under both diabetic and non-diabetic conditions. The diabetic mice had significantly higher levels of blood glucose compared with the non-diabetic mice (Fig. 1A). DMF did not alter the levels of blood glucose in either condition (Fig. 1A).
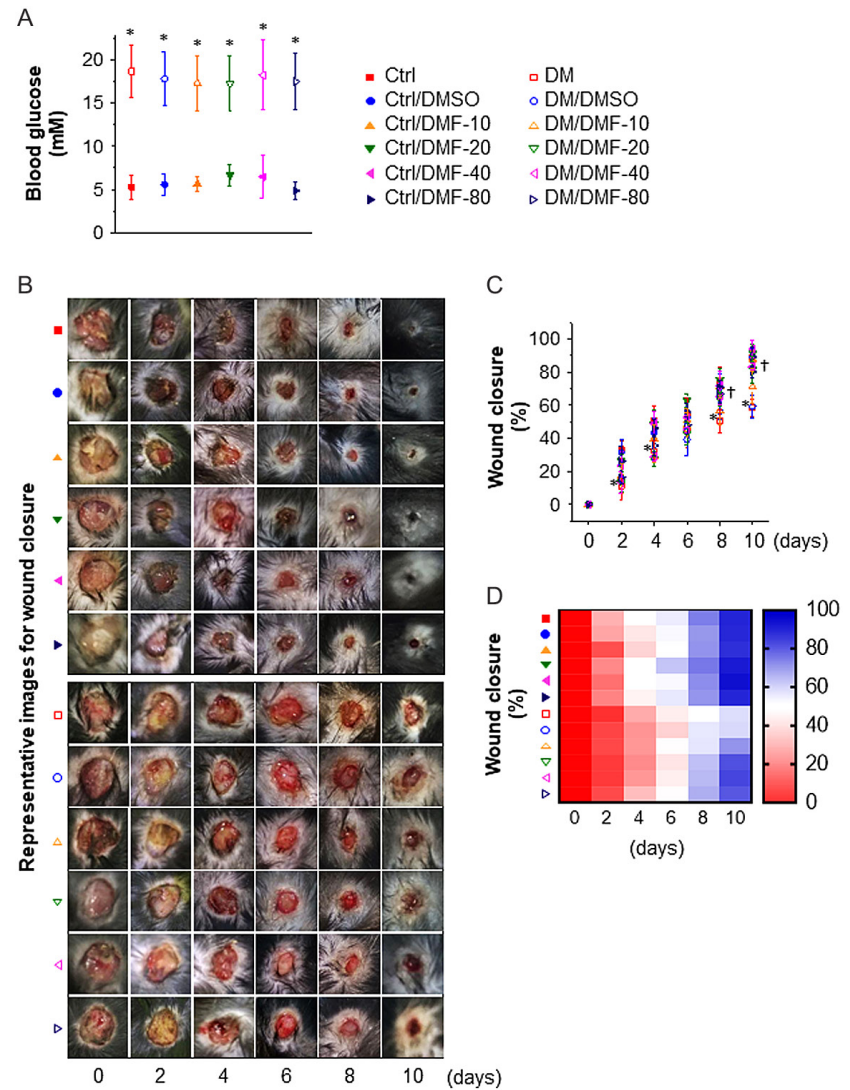

Figure 1

DMF accelerated wound closure under diabetic, but not non-diabetic conditions. (A) Blood glucose levels 1 week after the last injection of STZ. (B) Representative images of wound closure every other day, for a total period of 10 days after the onset of DM. (C) The percentage of wound closure was calculated. (D) Heat map of wound closure. The data are presented as means \pm S.D. $(n=6) .{ }^{*} P<0.05$ vs $C$ trl; ${ }^{\dagger} P<0.05$ vs DM. Symbols: red solid square, Ctrl; red hollow square, DM; blue solid circle, Ctrl/DMSO; blue hollow circle, DM/DMSO; orange solid up-triangle, Ctrl/DMF-10; orange hollow up-triangle, DM/DMF-10; green solid down-triangle, Ctrl/DMF-20; green hollow down-triangle, DM/DMF-20; magenta solid left-triangle, Ctrl/DMF-40; magenta hollow left-triangle, DM/DMF-40; navy solid right-triangle, Ctrl/DMF-80; navy hollow right-triangle, DM/DMF-80. Ctrl, control; DM, diabetes mellitus; DMF, dimethyl fumarate; DMF-10, DMF at $10 \mathrm{mg} / \mathrm{kg}$; DMF-20, DMF at $20 \mathrm{mg} / \mathrm{kg}$; DMF-40, DMF at $40 \mathrm{mg} / \mathrm{kg}$; DMF-80, DMF at $80 \mathrm{mg} / \mathrm{kg} ; \mathrm{STZ}$, streptozotocin. 
The percentage of wound closure was determined in all the mice every other day after the onset of DM (Fig. 1B) to directly evaluate the effect of DMF on wound healing. DM significantly delayed wound closure 2, 4, 8 and 10 days after the onset of DM (Fig. 1C, red hollow squares vs red solid squares). Although there was no significant difference between the percentages of wound closure 6 days after the onset of DM, DM resulted in a lower mean level compared with Ctrl (Fig. 1C, red hollow square vs red solid square). DM-delayed wound closure was rescued by DMF at $20 \mathrm{mg} / \mathrm{kg}$ (Fig. 1C, green hollow down-triangle), $40 \mathrm{mg} / \mathrm{kg}$ (Fig. 1C, magenta hollow lefttriangle) and $80 \mathrm{mg} / \mathrm{kg}$ (Fig. 1C, navy hollow right-triangle) 8 and 10 days after the onset of DM. The $10 \mathrm{mg} / \mathrm{kg}$ DMF (Fig. 1C, orange hollow up-triangle) dose did not induce a significant acceleration of wound closure in diabetic mice. Surprisingly, in contrast to the beneficial effect observed under diabetic conditions, DMF did not facilitate wound closure in non-diabetic mice, regardless of dosage (Fig. 1C, solid symbols). Figure 1D shows the effects of DMF on the diabetic wound healing also as a heat map.

\section{Cutaneous NRF2 was activated by DMF to a greater extent in diabetic mice compared with that in non-diabetic mice}

The following study evaluated the effect of DMF on cutaneous NRF2/HO1 signaling in both diabetic and nondiabetic mice (Fig. 2A, B and C). NRF2 protein, as well as $\mathrm{Ho1}$ mRNA and protein, were elevated under diabetic conditions (Fig. 2A, B and C, red hollow bars vs red solid bars), as a compensatory protective effect in response to DM. The $10 \mathrm{mg} / \mathrm{kg}$ dose of DMF enhanced the NRF2/HO1 signaling significantly under both conditions (Fig. 2A, $\mathrm{B}$ and $\mathrm{C}$, orange bars vs red bars). NRF2/HO1 signaling was enhanced by DMF more prominently at 20, 40 and $80 \mathrm{mg} / \mathrm{kg}$ compared with that observed at the $10 \mathrm{mg} / \mathrm{kg}$ dose (Fig. 2A, B and C, green, magenta and navy bars vs orange bars). Among all the doses of DMF tested in this study, $20 \mathrm{mg} / \mathrm{kg}$ was the lowest dose to activate cutaneous NRF2 signaling as effectively as the higher doses (Fig. 2A, $\mathrm{B}$ and $\mathrm{C}$ ). These results demonstrate the ability of DMF to activate cutaneous NRF2 in mice under both diabetic and non-diabetic conditions.

\section{DMF attenuated cutaneous oxidative damage and inflammation under diabetic conditions}

The cutaneous levels of MDA (Fig. 2D) along with the mRNA levels of Vcam-1 (Fig. 2E) and Icam-1 (Fig. 2F) were
A

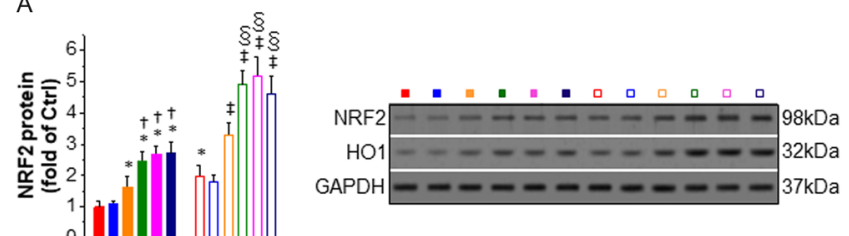

B

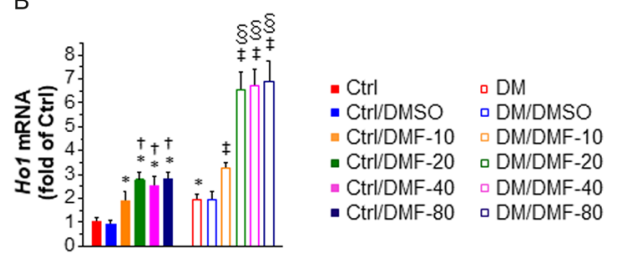

C

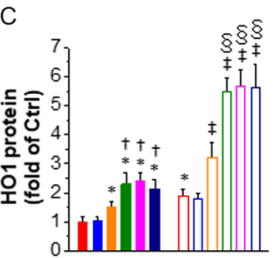

D

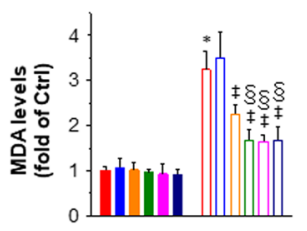

E

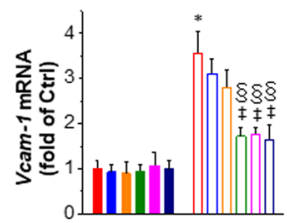

$\mathrm{F}$

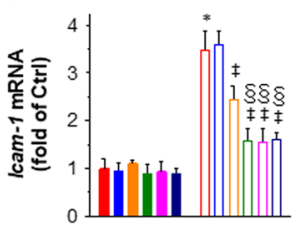

Figure 2

DMF attenuated cutaneous oxidative damage and inflammation in diabetic mice, but not in non-diabetic mice. (A) Western blotting was used to determine the levels of NRF2 protein. In addition, the expression of (B) Ho1 mRNA and (C) protein was measured using RT-PCR and Western blotting respectively, as a reflection of the function of NRF2. (D) A commercial kit was used to measure the levels of MDA, and RT-PCR was used to determine the mRNA levels of (E) Vcam-1 and (F) Icam-1. The data were normalized to $\mathrm{Ctrl}$ and presented as means \pm S.D. $(n=6)$. ${ }^{\star} P<0.05$ vs $C$ trl; ${ }^{+} P<0.05$ vs DMF-10; ${ }^{\ddagger} P<0.05$ vs DM; $\$ P<0.05$ vs DMF- 10 . Bars: red solid, Ctrl; red hollow, DM; blue solid, Ctrl/DMSO; blue hollow, DM/DMSO; orange solid, Ctrl/DMF-10; orange hollow, DM/DMF-10; green solid, Ctrl/DMF-20; green hollow, DM/DMF-20; magenta solid, Ctrl/ DMF-40; magenta hollow, DM/DMF-40; navy solid, Ctrl/DMF-80; navy hollow, DM/DMF-80. Ho1, heme oxygenase-1; Icam-1, intercellular adhesion molecule-1; MDA, malondialdehyde; NRF2, nuclear factor erythroid 2-related factor 2; Vcam-1, vascular cell adhesion molecule-1. Other abbreviations are the same as those in Fig. 1. A full colour version of this figure is available at https://doi.org/10.1530/JME-18-0102.

determined in diabetic and non-diabetic mice to assess the effect of DMF on wound healing at the molecular level. DMF attenuated all these parameters for oxidative damage (Fig. 2D) and inflammation (Fig. 2E and F) in the diabetic mice, with $20 \mathrm{mg} / \mathrm{kg}$ being the lowest dose to show similar effectiveness to that demonstrated by the higher doses (Fig. 2D, E and F, right panels). The DMF-attenuated cutaneous oxidative damage and inflammation under diabetic conditions (Fig. 2D, E and F, right panels) is in 
line with the DMF-enhanced cutaneous NRF2 antioxidant activity observed in diabetic mice (Fig. 2A, B and C, right panels).

Despite the significant enhancement of NRF2/HO1 signaling (Fig. 2A, B and C, left panels), and in accordance with the non-accelerated non-diabetic wound closure following treatment (Fig. 1B), DMF did not decrease the cutaneous levels of MDA and mRNA expression of Vcam1 and Icam-1 in non-diabetic mice, regardless of dosage (Fig. 2D, E and F, left panels). Thus, in the present study, NRF2 activation by DMF was shown to have distinct effects on wound healing, depending on the different levels of blood glucose. Oxidative stress is a main mechanism through which DM induces complications and NRF2 signaling is activated following DM-induced oxidative stress. Therefore, it was hypothesized that under normal levels of blood glucose, the skin produces low levels of free radicals as targets for the DMF-induced NRF2 antioxidant scavenging activity. This effect may lead to the invalid effect of DMF on the non-diabetic wound healing. This hypothesis was tested through the following studies.

\section{Oxidative damage, inflammation and NRF2 signaling exhibited prominently in keratinocytes}

Cutaneous morphological change was evaluated by H\&E staining, which did not show any obvious abnormality in the DM and DMF-treated $(20 \mathrm{mg} / \mathrm{kg})$ DM groups (Fig. 3A). IHC staining revealed that oxidative damage (4-HNE, Fig. 3B) and inflammation (VCAM-1, Fig. 3C) were preferably generated in keratinocytes and sebaceous gland under diabetic conditions. Keratinocytes actively responded to DMF that produced NRF2 (Fig. 3D) and HO1 (Fig. 3E), leading to a decrease in 4-HNE- and VCAM-1-positive areas (Fig. 3B and C). These results are consistent with previous reports highlighting the key role of keratinocytes in the diabetic wound healing ( $\mathrm{Hu} \& \mathrm{Lan}$ 2016, Long et al. 2016, 2017). Hence, the keratinocytes were culled in the following studies.

\section{NRF2 signaling was activated by $\mathrm{H}_{2} \mathrm{O}_{2}$ or $\mathrm{HG}$ to a similar extent}

Based on the findings in the diabetic and non-diabetic mice (Figs 1 and 2), it was hypothesized that a sufficient level of ROS (producing excessive free radicals for the antioxidant scavenging activity) is required for the protective effect of DMF-induced NRF2 activation on wound healing. This hypothesis was tested by subjecting keratinocytes to $\mathrm{H}_{2} \mathrm{O}_{2}$ or $\mathrm{HG}$, in the presence or absence of
DMF. The introduction of $\mathrm{H}_{2} \mathrm{O}_{2}$ may elucidate the role of ROS in determining DMF-induced protection. As Fig. 4A, B, C, D and E shows, DMF activated NRF2 signaling under the NG condition, which is in agreement with the findings observed in non-diabetic mice (Fig. 2A, B and C, left panels). The expression of NRF2 protein (Fig. 4A), along with the mRNA and protein levels of its target genes Ho1 and Nqo1 (Fig. 4B, C, D and E), were significantly enhanced following treatment with $\mathrm{H}_{2} \mathrm{O}_{2}$ or $\mathrm{HG}$ to similar extents. These effects were enhanced further by DMF (Fig. 4A, B, C, D and E). These results demonstrate that DMF, $\mathrm{H}_{2} \mathrm{O}_{2}$ and $\mathrm{HG}$ are capable of inducing NRF2 antioxidant signaling in keratinocytes.

\section{DMF alleviated oxidative damage and inflammation in keratinocytes following the generation of sufficient levels of ROS by $\mathrm{H}_{2} \mathrm{O}_{2}$ or HG}

The following study tested the effect of $\mathrm{DMF}, \mathrm{H}_{2} \mathrm{O}_{2}$ and $\mathrm{HG}$ on the generation of ROS (Fig. 5A), oxidative damage (Fig. 5B) and inflammation (Fig. 5C, D, E and F) in keratinocytes. Higher levels of ROS (Fig. 5A) and MDA (Fig. 5B), along with enhanced expression of Vcam-1 (Fig. 5C and D) and Icam-1 (Fig. 5E and F) were detected following treatment with $\mathrm{H}_{2} \mathrm{O}_{2}$ or $\mathrm{HG}$ compared with those recorded under the NG condition. Under the latter condition, DMF did not affect these parameters significantly (Fig. 5A, B, C, D, E and F), in spite of its significant effect on the induction of NRF2 signaling (Fig. 4A, B, C, D and E). However, following the drastic induction of ROS levels by $\mathrm{H}_{2} \mathrm{O}_{2}$ or $\mathrm{HG}$ (Fig. 5A), DMF attenuated the $\mathrm{H}_{2} \mathrm{O}_{2}$ - or $\mathrm{HG}$-induced oxidative damage and inflammation (Fig. 5B, C, D, E and F). HG produced ROS, MDA and inflammation to similar extents to those generated by $\mathrm{H}_{2} \mathrm{O}_{2}$ (Fig. $5 \mathrm{~A}, \mathrm{~B}, \mathrm{C}, \mathrm{D}, \mathrm{E}$ and F). Thus, $\mathrm{H}_{2} \mathrm{O}_{2}$ as an active form of ROS may account for the HG-induced oxidative damage and inflammation (Fig. 5B, C, D, E and F). These findings suggest that sufficient levels of ROS are required for the protective effect of DMF (Fig. 5B, C, D, E and F).

\section{Discussion}

The present study investigated the effect of DMF on the diabetic and non-diabetic wound healing. Cutaneous NRF2 antioxidant signaling was activated by DMF under both the diabetic and non-diabetic conditions. This effect accelerated wound healing in the diabetic mice; however, this was not observed in the non-diabetic mice. By increasing the levels of ROS in keratinocytes through 

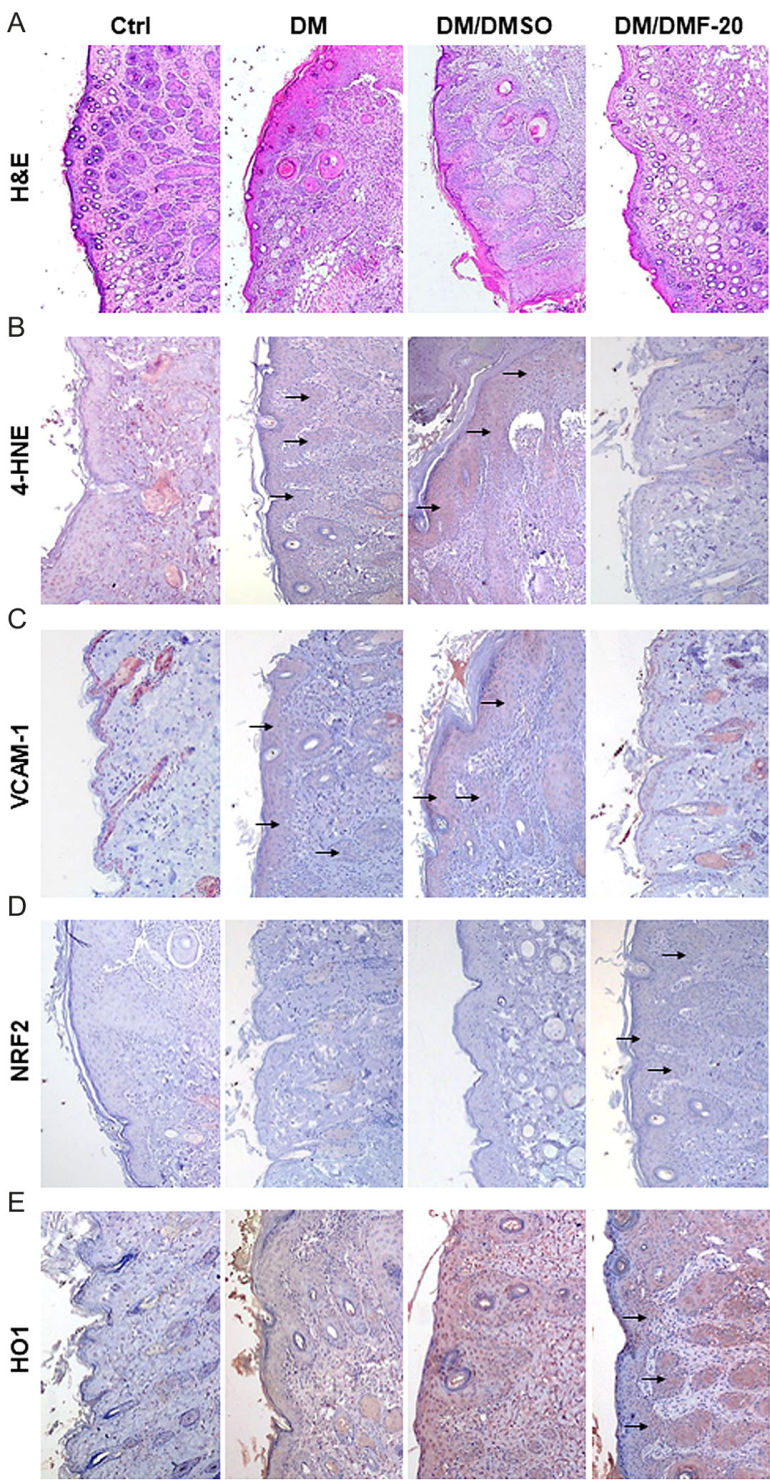

- Ctrl

- DM

- DM/DMSO

- DM/DMF-20
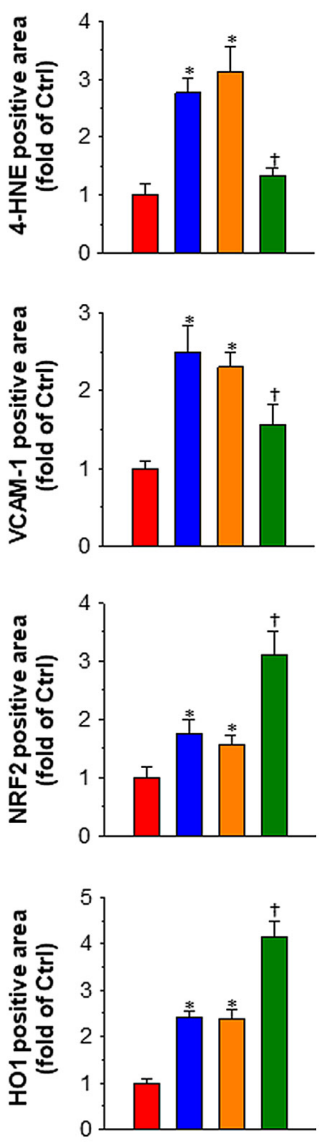

Figure 3

Oxidative damage, inflammation, and NRF2 signaling was exhibited prominently in keratinocytes. (A) H\&E staining was performed in the Ctrl, DM, and DMF-20 or vehicle-treated DM groups to evaluate morphology of the skin. IHC staining was performed using antibodies against (B) 4-HNE, (C) VCAM-1, (D) NRF2, and (E) HO1 to determine the location of oxidative damage, inflammation, and NRF2 signaling. Positively stained areas of the epidermis, excluding the sebaceous gland were quantified. Symbol: black arrow, positively stained keratinocytes. 4-HNE, 4-hydroxynonenal; IHC, immunohistochemical. Other abbreviations are the same as those in Figs 1 and 2. treatment with $\mathrm{H}_{2} \mathrm{O}_{2}$ or $\mathrm{HG}$, it was shown that sufficient levels of ROS are required for the protective effect of DMF.

The findings of the present study showed that ROS activate the effect of DMF on wound healing (Fig. 6). Specifically, under non-diabetic conditions, the wound expresses basal levels of ROS which in turn produce limited levels of free radicals (Fig. 6, the second quadrant). NRF2 antioxidant signaling is activated in response to ROS, scavenging the free radicals and leading to very limited oxidative damage and inflammation (Fig. 6, the second quadrant). Consequently, this effect facilitates normal wound healing (Fig. 6, the second quadrant). On the contrary, high levels of ROS are produced in DM, producing excessive levels of free radicals that exceed the scavenging capacity of NRF2 antioxidant signaling (Fig. 6, the third quadrant). This effect causes severe oxidative

\begin{tabular}{|lr} 
https://jme.bioscientifica.com & C 2018 Society for Endocrinology \\
https://doi.org/10.1530/JME-18-0102 & Published by Bioscientifica Ltd. \\
Printed in Great Britain
\end{tabular}

damage and inflammation which consequently impair the diabetic wound healing (Fig. 6, the third quadrant). When treated with DMF, NRF2 signaling is activated under both the diabetic (Fig. 6, the fourth quadrant) and non-diabetic conditions (Fig. 6, the first quadrant). Under diabetic conditions, DMF-elevated antioxidants neutralize ROS-induced excessive free radicals, minimizing oxidative damage and inflammation, and accelerating the diabetic wound healing (Fig. 6, the fourth quadrant). However, despite the increased levels of NRF2-dependent antioxidants, low levels of free radicals are induced by the basal ROS levels under non-diabetic conditions, providing insufficient targets for DMF-induced antioxidants (Fig. 6, the first quadrant). Collectively, the difference in cutaneous ROS levels between the diabetic and non-diabetic conditions may determine the DMF-induced protection. 
A
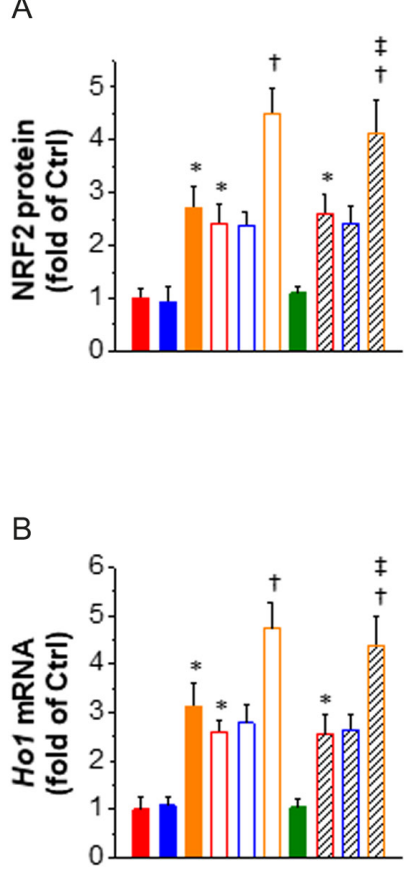

$\mathrm{D}$

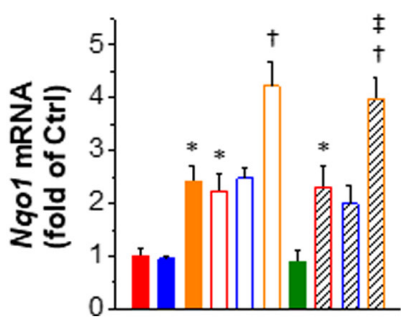

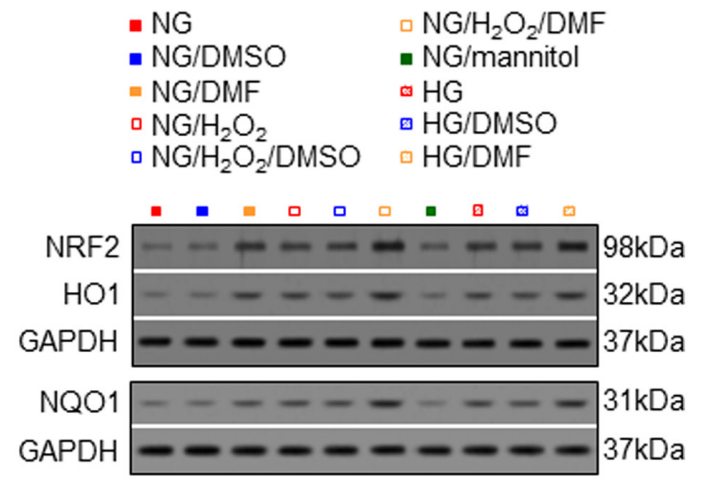

C

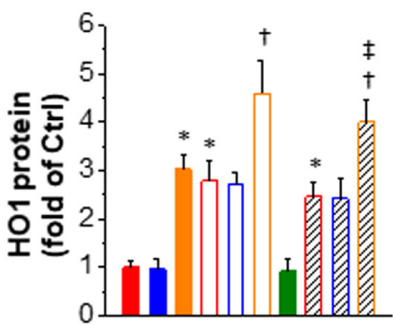

$E$

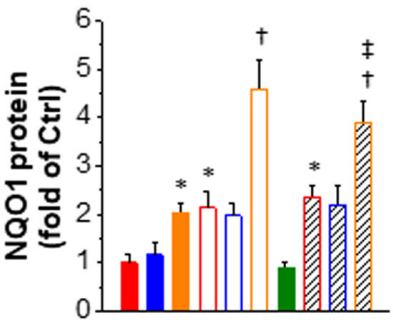

Figure 4

NRF2 signaling was activated by $\mathrm{H}_{2} \mathrm{O}_{2}$ or $\mathrm{HG}$ to a similar extent. Keratinocytes were subjected to $\mathrm{NG}, \mathrm{H}_{2} \mathrm{O}_{2}$, or $\mathrm{HG}$ in the presence or absence of DMF, to measure the levels of (A) NRF2 protein, and the mRNA and protein expression of ( $\mathrm{B}$ and C) Ho1, and (D and E) Nqo1. The data were normalized to NG and presented as means \pm S.D. $(n=3) .{ }^{*} P<0.05$ vs NG; ${ }^{+} P<0.05$ vs NG/DMF; ${ }^{\ddagger} P<0.05$ vs HG. Bars: red solid, NG; blue solid, NG/DMSO; orange solid, NG/DMF; red hollow, $\mathrm{NG} / \mathrm{H}_{2} \mathrm{O}_{2}$; blue hollow, $\mathrm{NG} / \mathrm{H}_{2} \mathrm{O}_{2} / \mathrm{DMSO}$; orange hollow, $\mathrm{NG} / \mathrm{H}_{2} \mathrm{O}_{2} / \mathrm{DMF}$; green solid, $\mathrm{NG}$ /mannitol; red hollow with diagonal stripes, HG; blue hollow with diagonal stripes, $\mathrm{HG} / \mathrm{DMSO}$; orange hollow with diagonal stripes, $\mathrm{HG} / \mathrm{DMF} . \mathrm{H}_{2} \mathrm{O}_{2}$, hydrogen peroxide; $\mathrm{HG}$, high glucose; $\mathrm{NG}$, normal glucose; Nqo1, NAD(P)H dehydrogenase quinone 1; ROS, reactive oxygen species. Other abbreviations are the same as those in Figs 1 and 2. A full colour version of this figure is available at https://doi. org/10.1530/JME-18-0102.
In numerous studies, the activation of NRF2 under normal conditions did not decrease oxidative stress and inflammation, in contrast to the benefits of NRF2 activation observed under diabetic conditions (Wang et al. 2014, Wu et al. 2015, Gu et al. 2016, Dong et al. 2017, Pan et al. 2017, Sun et al. 2017). Different from these cases, the non-diabetic wound healing is a pathophysiological procedure, rather than a normal condition. Basal levels of ROS trigger NRF2 signaling and accelerate the wound healing process (Roy et al. 2006, D'Autreaux \& Toledano 2007, Dunnill et al. 2017), as a compensatory mechanism of self-defense in response to trauma. However, these basal levels of ROS produced by non-diabetic wounds are insufficient to produce enough free radicals for DMFinduced antioxidants to scavenge upon. In contrast, following the generation of free radicals by DM exceeding the cellular self-defense capacity, supplementation of more antioxidants becomes an urgent requirement. This provides insight into the potential future use of DMF for the treatment of the diabetic non-healing wound, but not the non-diabetic wound. Furthermore, the finding that ROS activates DMF-induced protection may provide a basis for the potential use of DMF in several diseases, with exceptionally enhanced oxidative stress playing a key role.

DM affects the entire body, with multiple organs and systems involved. Common diabetic complications, in addition to the diabetic non-healing wound (Long et al. 2016), include cardiovascular disease (Beckman et al. 2002, Wu et al. 2018), nephropathy (Wu et al. $2014 a$ ), retinopathy and neuropathy (Volpe et al. 2018). Oxidative stress is the main mechanism through which DM induces complications. By targeting oxidative stress, the activation of NRF2 was shown to be effective against all the aforementioned diabetic complications (Negi et al. 2011, Zheng et al. 2011, Deliyanti et al. 2016, Gu et al. 2016, Xie et al. 2016). Thus, beneficial effects on multiple organs may be exerted through the activation of NRF2 under DM, granting a unique advantage in the management of DM and its complications. 
A

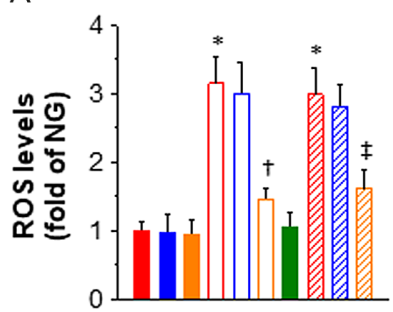

C

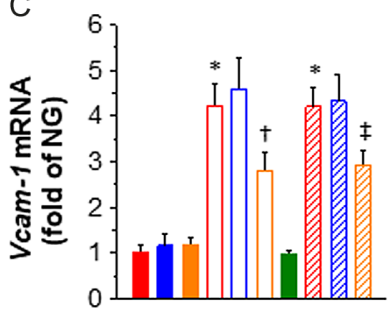

E

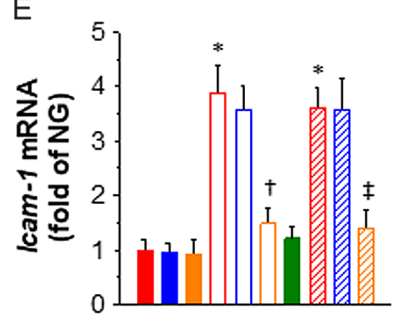

\section{- NG}

- NG/DMSO

- NG/DMF

$\square \mathrm{NG} / \mathrm{H} 2 \mathrm{O} 2$

- NG/H2O2/DMSO
B

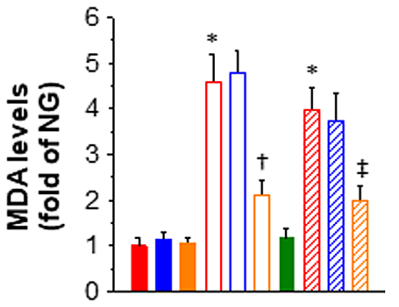

D

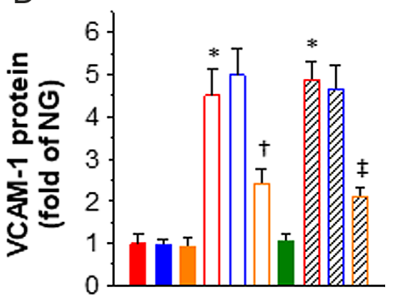

F

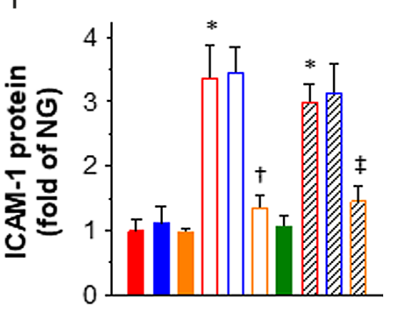

- NG/H2O2/DMF

- NG/Mannitol

๑ $\mathrm{HG}$

- HG/DMSO

- HG/DMF

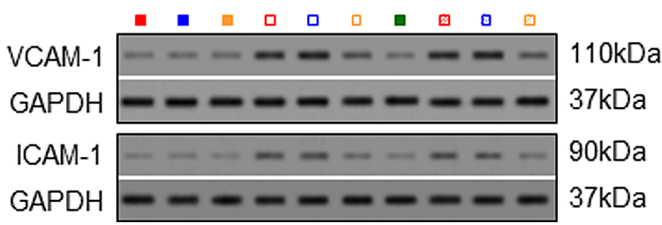

Figure 5

DMF alleviated oxidative damage and inflammation in keratinocytes following the generation of sufficient levels of ROS by $\mathrm{H}_{2} \mathrm{O}_{2}$ or HG. Keratinocytes were treated with DMF or vehicle, in the presence or absence of $\mathrm{H}_{2} \mathrm{O}_{2}$ or HG. The levels of (A) ROS and (B) MDA, along with the mRNA and protein expression of ( $C$ and $D) V c a m-1$ and ( $E$ and $F$ ) Icam-1 were determined. The data were normalized to NG and was presented as means \pm S.D. $(n=3)$. ${ }^{*} P<0.05$ vs NG; ${ }^{+} P<0.05$ vs NG/H $\mathrm{O}_{2}$; ${ }^{\ddagger} P<0.05$ vs HG. Bars are the same as those in Fig. 4 . Abbreviations are the same as those in Figs 1, 2 and 4. A full colour version of this figure is available at https://doi.org/10.1530/JME-18-0102.

To date, two known activators of NRF2, namely SFN and CA, were shown to be effective against the diabetic wound healing in a study using a mouse model (Long et al. 2016). In the present study, DMF was shown to accelerate this procedure, in a similar manner to SFN and CA. An advantage of DMF is safety as evidenced by the Printed in Great Britain

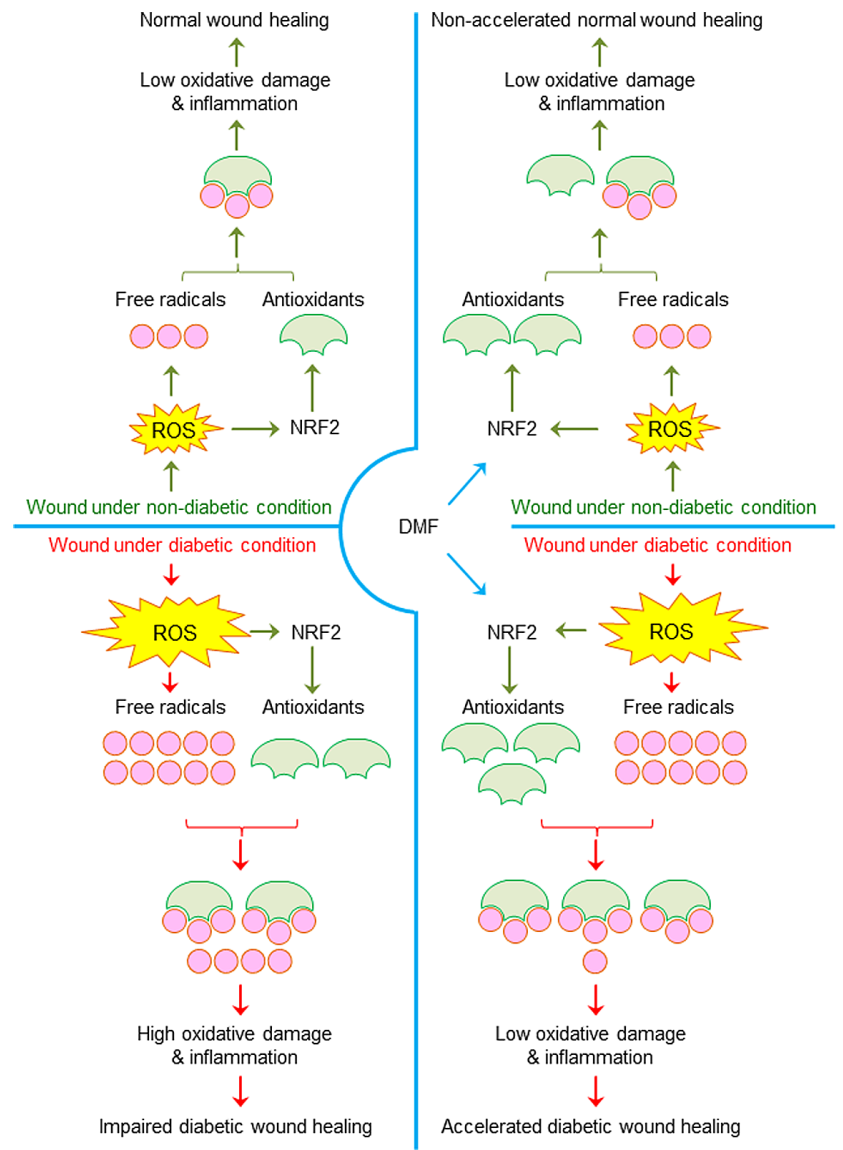

Figure 6

Possible role of ROS in mediating the protection offered by DMF. Under non-diabetic conditions, the wound expresses basal levels of ROS, producing limited levels of free radicals. NRF2 antioxidant signaling is activated in response to basal ROS, scavenging the free radicals and leading to mitigated oxidative damage and inflammation. This mild ROS-induced activation of NRF2 facilitates normal wound healing. On the contrary, under diabetic conditions, ROS are abundantly produced, generating excessive free radicals that exceed the scavenging capacity of NRF2 antioxidant signaling. This effect causes severe oxidative damage and inflammation which impair diabetic wound healing. When treated with DMF, NRF2 signaling is activated under both the diabetic and non-diabetic conditions. Under diabetic conditions, the DMF-elevated antioxidants neutralize ROS-induced excessive free radicals, attenuating oxidative damage and inflammation and accelerating diabetic wound healing. However, under non-diabetic conditions, low levels of free radicals are induced by the lower levels of ROS. This provides insufficient targets for DMF-induced antioxidants. This may explain the nonaccelerated wound healing process in the presence of DMF under non-diabetic conditions. Symbols: $\downarrow$, activation; $\perp$, inhibition. Abbreviations are the same as those in Figs 1, 2 and 4. A full colour version of this figure is available at https://doi.org/10.1530/JME-18-0102.

approval of its clinical use in treatment of MS (Linker \& Gold 2013, Bomprezzi 2015). The present study is the first to unveil the effectiveness of DMF on the diabetic wound healing, in addition to its known ability to improve MS.

Collectively, the present study demonstrates for the first time that DMF improves wound healing under 
diabetic conditions, although this effect was not observed in non-diabetic conditions. These findings reveal an indispensable role of ROS in mediating the effect of DMF on wound healing, and a new therapeutic effect of DMF, which may attract attention to ROS in the future for the management of diseases involving activators of NRF2.

\section{Declaration of interest}

The authors declare that there is no conflict of interest that could be perceived as prejudicing the impartiality of the research reported.

\section{Funding}

This work was supported in part by the National Natural Science Foundation of China (81600573) and Norman Bethune Program of Jilin University (2015438) to Hao Wu; Natural Science Foundation of Jilin Province (JJKH20180362KJ) to Ying Li and Natural Science Foundation of Jilin Province (2018SCZWSZX-045) to Ziping Jiang.

\section{Author contribution statement}

Hao Wu conceived the idea. Hao Wu, Ziping Jiang, Ying Li, Fuzhe Ma and Yuguo Song designed the research. Ying Li, Fuzhe Ma, Huimin Li, Yuguo Song, Huan Zhang, Ziping Jiang and Hao Wu researched and interpreted the data and were involved in the discussion. Hao Wu, Ziping Jiang and Ying Li wrote the manuscript. Ying Li, Fuzhe Ma, Huimin Li, Yuguo Song, Huan Zhang, Ziping Jiang and Hao Wu reviewed and revised the manuscript. Hao Wu, Ying $\mathrm{Li}$ and Ziping Jiang provided funding. All authors approve the version to be published.

\section{References}

Beckman JA, Creager MA \& Libby P 2002 Diabetes and atherosclerosis: epidemiology, pathophysiology, and management. JAMA 287 2570-2581. (https://doi.org/10.1001/jama.287.19.2570)

Bomprezzi R 2015 Dimethyl fumarate in the treatment of relapsingremitting multiple sclerosis: an overview. Therapeutic Advances in Neurological Disorders 8 20-30. (https://doi. org/10.1177/1756285614564152)

D'Autreaux B \& Toledano MB 2007 ROS as signalling molecules: mechanisms that generate specificity in ROS homeostasis. Nature Reviews Molecular Cell Biology 8 813-824. (https://doi.org/10.1038/nrm2256)

Davis FM, Kimball A, Boniakowski A \& Gallagher K 2018 Dysfunctional wound healing in diabetic foot ulcers: new crossroads. Current Diabetes Reports 18 2. (https://doi.org/10.1007/s11892-018-0970-z)

Deliyanti D, Lee JY, Petratos S, Meyer CJ, Ward KW, Wilkinson-Berka JL \& de Haan JB 2016 A potent Nrf2 activator, dh404, bolsters antioxidant capacity in glial cells and attenuates ischaemic retinopathy. Clinical Science 130 1375-1387. (https://doi. org/10.1042/CS20160068)

Dong W, Jia Y, Liu X, Zhang H, Li T, Huang W, Chen X, Wang F, Sun W \& Wu H 2017 Sodium butyrate activates NRF2 to ameliorate diabetic nephropathy possibly via inhibition of HDAC. Journal of Endocrinology 232 71-83. (https://doi.org/10.1530/JOE-16-0322)

Dunnill C, Patton T, Brennan J, Barrett J, Dryden M, Cooke J, Leaper D \& Georgopoulos NT 2017 Reactive oxygen species (ROS) and wound healing: the functional role of ROS and emerging ROS-modulating technologies for augmentation of the healing process. International Wound Journal 14 89-96. (https://doi.org/10.1111/iwj.12557)
Garstkiewicz M, Strittmatter GE, Grossi S, Sand J, Fenini G, Werner S, French LE \& Beer HD 2017 Opposing effects of Nrf2 and Nrf2activating compounds on the NLRP3 inflammasome independent of Nrf2-mediated gene expression. European Journal of Immunology 47 806-817. (https://doi.org/10.1002/eji.201646665)

Gu J, Cheng Y, Wu H, Kong L, Wang S, Xu Z, Zhang Z, Tan Y, Keller BB, Zhou H, et al. 2016 Metallothionein is downstream of Nrf2 and partially mediates sulforaphane prevention of diabetic cardiomyopathy. Diabetes 66 529-542. (https://doi.org/10.2337/ db15-1274)

Hu SC \& Lan CE 2016 High-glucose environment disturbs the physiologic functions of keratinocytes: focusing on diabetic wound healing. Journal of Dermatological Science 84 121-127. (https://doi. org/10.1016/j.jdermsci.2016.07.008)

Hulsmans M \& Holvoet P 2010 The vicious circle between oxidative stress and inflammation in atherosclerosis. Journal of Cellular and Molecular Medicine 14 70-78. (https://doi. org/10.1111/j.1582-4934.2009.00978.x)

Kampfer H, Schmidt R, Geisslinger G, Pfeilschifter J \& Frank S 2005 Wound inflammation in diabetic ob/ob mice: functional coupling of prostaglandin biosynthesis to cyclooxygenase-1 activity in diabetesimpaired wound healing. Diabetes 54 1543-1551. (https://doi. org/10.2337/diabetes.54.5.1543)

Kaspar JW, Niture SK \& Jaiswal AK 2009 Nrf2:INrf2 (Keap1) signaling in oxidative stress. Free Radical Biology and Medicine 47 1304-1309. (https://doi.org/10.1016/j.freeradbiomed.2009.07.035)

Kato M, Wang M, Chen Z, Bhatt K, Oh HJ, Lanting L, Deshpande S, Jia Y, Lai JY, O'Connor CL, et al. 2016 An endoplasmic reticulum stress-regulated lncRNA hosting a microRNA megacluster induces early features of diabetic nephropathy. Nature Communication $\mathbf{7}$ 12864. (https://doi.org/10.1038/ncomms12864)

Lan CC, Wu CS, Huang SM, Wu IH \& Chen GS 2013 High-glucose environment enhanced oxidative stress and increased interleukin-8 secretion from keratinocytes: new insights into impaired diabetic wound healing. Diabetes 62 2530-2538. (https://doi.org/10.2337/ db12-1714)

Lee YJ, Bae JH, Kang SG, Cho SW, Chun DI, Nam SM, Kim CH, Nam HS, Lee SH, Lee SH, et al. 2017 Pro-oxidant status and Nrf2 levels in psoriasis vulgaris skin tissues and dimethyl fumarate-treated HaCaT cells. Archives of Pharmacal Research 40 1105-1116. (https://doi. org/10.1007/s12272-017-0955-5)

Linker RA \& Gold R 2013 Dimethyl fumarate for treatment of multiple sclerosis: mechanism of action, effectiveness, and side effects. Current Neurology and Neuroscience Reports 13 394. (https://doi.org/10.1007/ s11910-013-0394-8)

Long M, Rojo de la Vega M, Wen Q, Bharara M, Jiang T, Zhang R, Zhou S, Wong PK, Wondrak GT, Zheng H, et al. 2016 An essential role of NRF2 in diabetic wound healing. Diabetes 65 780-793. (https://doi.org/10.2337/db15-0564)

Long M, Cai L, Li W, Zhang L, Guo S, Zhang R, Zheng Y, Liu X, Wang M, Zhou X, et al. 2017 DPP-4 inhibitors improve diabetic wound healing via direct and indirect promotion of epithelialmesenchymal transition and reduction of scarring. Diabetes $\mathbf{6 7}$ 518-531. (https://doi.org/10.2337/db17-0934)

Negi G, Kumar A \& Sharma SS 2011 Nrf2 and NF-kappaB modulation by sulforaphane counteracts multiple manifestations of diabetic neuropathy in rats and high glucose-induced changes. Current Neurovascular Research 8 294-304. (https://doi. org/10.2174/156720211798120972)

Pan C, Zhou S, Wu J, Liu L, Song Y, Li T, Ha L, Liu X, Wang F, Tian J, et al. 2017 NRF2 plays a critical role in both self and EGCG protection against diabetic testicular damage. Oxidative Medicine and Cellular Longevity 2017 3172692. (https://doi. org/10.1155/2017/3172692)

Pi J, Qu W, Reece JM, Kumagai Y \& Waalkes MP 2003 Transcription factor Nrf2 activation by inorganic arsenic in cultured keratinocytes: 
involvement of hydrogen peroxide. Experimental Cell Research 290 234-245. (https://doi.org/10.1016/S0014-4827(03)00341-0)

Roy S, Khanna S, Nallu K, Hunt TK \& Sen CK 2006 Dermal wound healing is subject to redox control. Molecular Therapy 13 211-220. (https://doi.org/10.1016/j.ymthe.2005.07.684)

Ruiz S, Pergola PE, Zager RA \& Vaziri ND 2013 Targeting the transcription factor Nrf2 to ameliorate oxidative stress and inflammation in chronic kidney disease. Kidney International $\mathbf{8 3}$ 1029-1041. (https://doi.org/10.1038/ki.2012.439)

Sun W, Liu X, Zhang H, Song Y, Li T, Liu X, Liu Y, Guo L, Wang F, Yang T, et al. 2017 Epigallocatechin gallate upregulates NRF2 to prevent diabetic nephropathy via disabling KEAP1. Free Radical Biology and Medicine 108 840-857. (https://doi.org/10.1016/j. freeradbiomed.2017.04.365)

Volpe CMO, Villar-Delfino PH, Dos Anjos PMF \& Nogueira-Machado JA 2018 Cellular death, reactive oxygen species (ROS) and diabetic complications. Cell Death and Disease 9 119. (https://doi. org/10.1038/s41419-017-0135-z)

Wang Y, Zhang Z, Guo W, Sun W, Miao X, Wu H, Cong X, Wintergerst KA, Kong X \& Cai L 2014 Sulforaphane reduction of testicular apoptotic cell death in diabetic mice is associated with the upregulation of Nrf2 expression and function. American Journal of Physiology: Endocrinology and Metabolism 307 E14-E23. (https://doi. org/10.1152/ajpcell.00270.2014)

Wu H, Kong L, Zhou S, Cui W, Xu F, Luo M, Li X, Tan Y \& Miao L 2014a The role of microRNAs in diabetic nephropathy. Journal of Diabetes Research 2014 920134. (https://doi.org/10.1155/2014/920134)
Wu H, Zhou S, Kong L, Chen J, Feng W, Cai J, Miao L \& Tan Y 2014b Metallothionein deletion exacerbates intermittent hypoxia-induced renal injury in mice. Toxicology Letters 232 340-348. (https://doi. org/10.1016/j.toxlet.2014.11.015)

Wu H, Kong L, Cheng Y, Zhang Z, Wang Y, Luo M, Tan Y, Chen X, Miao L \& Cai L 2015 Metallothionein plays a prominent role in the prevention of diabetic nephropathy by sulforaphane via up-regulation of Nrf2. Free Radical Biology and Medicine 89 431-442. (https://doi.org/10.1016/j.freeradbiomed.2015.08.009)

Wu H, Kong L, Tan Y, Epstein PN, Zeng J, Gu J, Liang G, Kong M, Chen X, Miao L, et al. 2016 C66 ameliorates diabetic nephropathy in mice by both upregulating NRF2 function via increase in miR200a and inhibiting miR-21. Diabetologia 59 1558-1568. (https://doi. org/10.1007/s00125-016-3958-8)

Wu H, Wu J, Zhou S, Huang W, Li Y, Zhang H, Wang J \& Jia Y 2018 SRT2104 attenuates diabetes-induced aortic endothelial dysfunction via inhibition of P53. Journal of Endocrinology 237 1-14. (https://doi. org/10.1530/JOE-17-0672)

Xie L, Gu Y, Wen M, Zhao S, Wang W, Ma Y, Meng G, Han Y, Wang Y, Liu G, et al. 2016 Hydrogen sulfide induces Keap1 S-sulfhydration and suppresses diabetes-accelerated atherosclerosis via Nrf2 activation. Diabetes 65 3171-3184. (https://doi.org/10.2337/db160020)

Zheng H, Whitman SA, Wu W, Wondrak GT, Wong PK, Fang D \& Zhang DD 2011 Therapeutic potential of Nrf2 activators in streptozotocin-induced diabetic nephropathy. Diabetes $\mathbf{6 0}$ 3055-3066. (https://doi.org/10.2337/db11-0807)

Received in final form 11 July 2018

Accepted 23 July 2018

Accepted Preprint published online 23 July 2018 (c) 2018 Society for Endocrinology Published by Bioscientifica Ltd. Printed in Great Britain 